\title{
Pensión esencial en Colombia. Aspectos a tener en cuenta en una futura reforma*
}

\section{Essential pension in Colombia. Considerations for a future reform}

\section{$\{\text { Mauricio Amador }\}^{\star \star}$}

Recibido: 5 de diciembre de 20I9. Aceptado: 5 de mayo de 2020. DoI: https://doi.org/IO.I860I/25390406. n5.04

Administrador de Empresas de la Pontificia Universidad Javeriana. Especialista en Análisis Financiero del Chase Manhattan Bank. Realizó el curso de Alta Gerencia de la Universidad Externado de Colombia. Cursó el programa de Desarrollo Directivo de inalde, Universidad de la Sabana. Ha sido presidente de Davivir S. A. y de Pensiones y Cesantías Santander S. A., Vicepresidente Financiero y de Planeación de Seguros Colmena y de Seguros del Comercio, y como Vicepresidente de la Titularizadora Colombiana. Se ha desempeñado como consultor de Seguridad Social y de los sectores financiero y de infraestructura. 


\section{Resumen}

El alto porcentaje de personas con edades superiores a los 65 años en Latinoamérica, que no tienen un ingreso asegurado, obliga a pensar en un mecanismo que solucione este problema, pero que a la vez reconozca el esfuerzo que hagan las personas por cotizar durante su etapa productiva. Es por ello que, frente a la posibilidad de que este tema se aborde en una futura reforma, que ojalá sea de protección integral a la vejez y no se limite a una pensional, se plantean los principales elementos que a mi juicio deberían ser tenidos en cuenta en dicha reforma.

\section{Palabras clave}

Esquema de pensión esencial, Colombia, desarrollo, implementación, administración, educación.

\section{Abstract}

The high percentage of people over the age of 65 , who do not have a guaranteed income is one of the highest in Latin America, forces us to think of a mechanism that solves this problem, but at the same time recognizes the effort they make people to quote during their productive stage. That is why, in the face of the possibility that this issue is addressed in a future reform, that hopefully is of integral protection to old age and is not limited to a pension, the main elements that in my opinion should be considered in account in said reform.

\section{KEYWORDS}

Essential pension, Colombia, development, implementation, administration, education.

\section{INTRODUCCIÓN}

Cuando se inicia el debate sobre el alcance que debe tener una reforma del sistema de pensiones, uno de los aspectos que siempre hace parte de la discusión es el de determinar si su objetivo es tener un sistema de pensiones, de base económica, o un sistema de protección integral a la vejez. Nosotros, los que estamos participando en la elaboración de este documento, consideramos que el objetivo debe centrarse en la opción de protección integral y ello hace obligatorio abordar el tema de cómo lograr que la persona que llega a la edad en que se le dificulta trabajar pueda obtener un ingreso mínimo para vivir. Por esto, quise dirigir parte de mi trabajo a explorar las alternativas existentes para garantizar dicho ingreso. 
Dentro de las reformas que se han realizado en diferentes países del mundo, incluida Latinoamérica, se han definido diversos esquemas para lograr este objetivo. Para efectos de este documento, todos estos se agruparán bajo el concepto de pensión esencial, dentro del cual se incluirán: (i) las pensiones mínimas garantizadas (PMG), (ii) las pensiones sociales o también llamadas asistenciales o de sobrevivencia, (iii) las pensiones básicas y (iv) los planes voluntarios de ahorro con subsidio del Estado

A pesar de tener un objetivo común, estos esquemas difieren en sus características. Uno de los principales aspectos a tener en cuenta en relación con cada uno es el origen de su financiación. De acuerdo con esto, podrían clasificarse en contributivos, si los recursos son aportados por los afiliados o las empresas; no contributivos, si provienen del presupuesto del Estado; o mixto si provienen de aportes de los afiliados, pero también tienen aportes del Estado.

Colombia está pensando iniciar una reforma de su Sistema General de Pensiones en un futuro cercano. Uno de los elementos a considerar será el posible desarrollo de un sistema de pilares en los que pueden concurrir en un mismo sistema y de forma integral esquemas no contributivos y contributivos (obligatorios o voluntarios). Como lo ha señalado el Banco Mundial en su informe Soporte del ingreso en la vejez en el siglo veintiuno (2005) ${ }^{\mathrm{I}}$, la implementación de un esquema de pensión esencial, con el cual se garantice un ingreso a la población que no está en condiciones de trabajar, sería indispensable para la constitución de los llamados Pilar cero y Pilar i. En este contexto, considero fundamental avanzar en el análisis sobre los principales aspectos que podría cobijar una pensión esencial en el país, así como los puntos críticos que deben ser tenidos en cuenta en relación con este tema, si se quiere hacer una reforma integral de la seguridad social en Colombia.

De acuerdo con lo anterior, este documento se divide en cuatro partes. En la primera, se presentarán brevemente los motivos por los cuales se considera adecuado implementar un esquema de protección a la vejez a través de una pensión esencial. En la segunda, se describirán los tipos de pensión esencial que se han establecido en algunas partes del mundo, con énfasis en Latinoamérica, considerando aspectos como tipo de pensión, nivel de cobertura y algunas características de la forma en que se han establecido. A partir de esto, se propondrá una clasificación de los esquemas existentes. En la tercera, se hará una breve descripción del sistema actual. A partir de allí, se hará énfasis

Robert Holzmann y Richard Hinz, Una perspectiva internacional de los sistemas de pensiones y de sus reformas (Banco mundial y Mayol Ediciones: Bogotá, 2005), https://documentsi.worldbank. org/curated/en/27 I 22 I 4683 15291697/pdf/Una-perspectiva-internacional-de-los-sistemas-depensiones-y-de-sus-reformas-Soporte-del-ingreso-economico-en-la-vejez-en-el-siglo-xxI.pdf 
en los aspectos críticos que deberían ser tenidos en cuenta para hacer viable la inclusión o modificación de la pensión esencial en el sistema colombiano y se plantearán las principales inquietudes que pueden surgir en el desarrollo de una reforma de este tipo. Y, finalmente, en la cuarta, se mostrará de manera sucinta una selección de los aspectos, tratados en el documento, que puedan tener una mayor relevancia si en una reforma del sistema pensional se va a discutir el establecimiento de una pensión esencial.

\section{¿Por qué considerar un esquema de pensión esencial en Colombia?}

Como se mencionó, ante la perspectiva de una reforma pensional en el país, es necesario que esta se dirija a lograr una protección integral de la vejez. Lo anterior teniendo en cuenta que, como lo señalaron en el 2010 el Banco Interamericano de Desarrollo (BID) y la Organización para la Cooperación y el Desarrollo Económico (oCDE), Colombia es el país latinoamericano con mayor porcentaje de población que supera los 65 años y que se encuentra en situación de pobreza. Además, es de gran preocupación que, según se muestra en el gráfico I a continuación, este porcentaje es considerablemente mayor para este grupo demográfico que para la población general del país.

\section{Gráfico 1.}

Tasas de POBReza total nacional y POBLación de 65 años 0 más.

Latinoamérica 2010. (Pobreza media con estándares INTERNACIONALES DE PPP 2,5 USD DIARIOS)

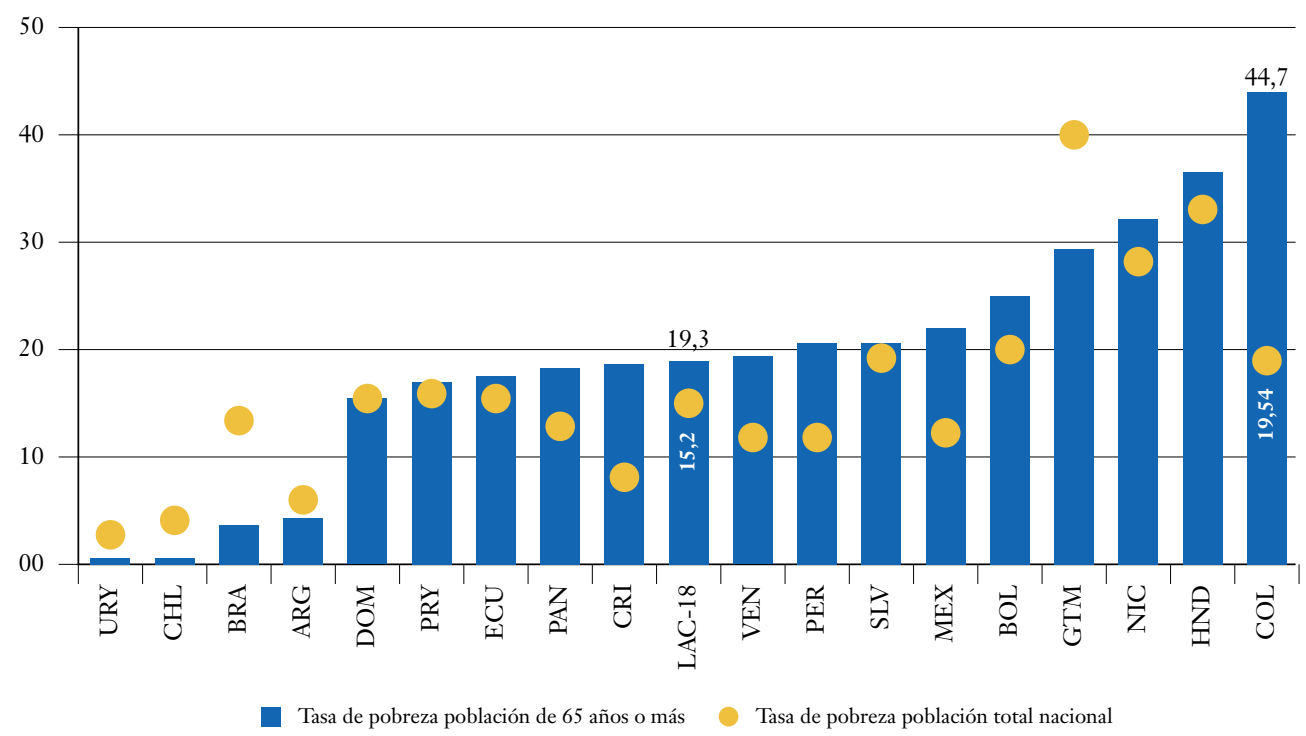

Fuente: Fedesarrollo y Fundación Saldarriaga Concha, 2015, p. I15 
De acuerdo con esto, las pensiones esenciales, especialmente aquellas que pueden caber en la definición de pensiones asistenciales, deben tener como objetivo primordial lograr sacar al mayor número de personas mayores del nivel de pobreza y de indigencia.

Podría pensarse que el derecho a una pensión debe ser resultado del esfuerzo que los afiliados hagan para realizar aportes a la seguridad social de forma obligatoria o voluntaria. Sin embargo, esta premisa debe ser puesta en discusión en países como Colombia en los cuales no hay un nivel adecuado de distribución de los ingresos (ni siquiera a través de los impuestos) y hay una dificultad grande para ahorrar dada la informalidad laboral, la necesidad de cubrir otras necesidades que tienen una mayor prioridad, la alta evasión de los empleadores en las cotizaciones lo mismo que en aquellos independientes con capacidad económica, la alta incidencia de empresas familiares o con imposibilidad de cubrir los costos laborales y la baja densidad en las cotizaciones entre otras. Por esto, es necesario considerar que, al menos durante las próximas décadas, el Estado debe ayudar a las familias para evitar que caigan en situación de pobreza cuando ya no estén en condiciones de trabajar.

Teniendo en cuenta la deseabilidad de desarrollar en el contexto colombiano un esquema de pensión esencial que permita mejorar los ingresos de la población mayor de 65 años, es pertinente explorar ahora las alternativas que existen para su implementación y que se consideran de utilidad para informar la toma de decisión sobre una futura reforma en Colombia.

\section{Esquemas de Pensión esencial}

\section{Desarrollo de pensiones esenciales}

A continuación, se presentan los casos de algunos países en los cuales se han implementado lo que hemos llamado de manera general esquemas de pensión esencial y que me permito tipificarlos.

Para ello, primero se muestra un cuadro resumen de las características principales de cada experiencia y posteriormente se describen con más detalle dichas características y su financiación contributiva, no contributiva o mixta, con el fin de tener una visión general de algunos modelos existentes. 
Cuadro 1.

Tipos de pensión ESENCial en algunos países

\begin{tabular}{|c|c|c|c|c|c|c|c|}
\hline \multirow{2}{*}{ País } & \multicolumn{7}{|c|}{ Tipo de pensión } \\
\hline & PMG & $\mathbf{P A}$ & PB & PAV & C & NC & $\mathbf{M x}$ \\
\hline Dinamarca & & $\mathrm{X}$ & $X$ & & PB & PA & \\
\hline Chile & $X$ & $X$ & $X$ & & & & PMG, РB \\
\hline Argentina & & $\mathrm{X}$ & & & & $\mathrm{PA}$ & \\
\hline Uruguay & & $\mathrm{X}$ & $\mathrm{X}$ & & Рв & $\mathrm{PA}$ & \\
\hline Bolivia & & $\mathrm{X}$ & $\mathrm{X}$ & & & $\mathrm{PA}$ & РВ \\
\hline Brasil & & $\mathrm{X}$ & & & & PA & \\
\hline
\end{tabular}

PMG: Pensión mínima garantizada, PA: Pensión asistencial o sobrevivencia, PB: Pensión básica, PAv: Plan de ahorro voluntario con subsidio del Estado.

c: Contributivo, Nc: No contributivo, Mx: Mixto.

Fuente: elaboración del autor.

a) Dinamarca: en el caso danés existe una clara diferenciación entre tres tipos de pensión. La primera es de carácter asistencial o de subsistencia no contributivo. Solo se otorga en casos extremos y está dirigida a las personas que demuestren que no cuentan con un ingreso individual o familiar.

El segundo tipo consiste en una prestación que se entrega a todas las personas que hayan trabajado un número mínimo de años y hayan cotizado. Consiste en un porcentaje de su salario promedio en su vida laboral.

Existe, finalmente, un tercer tipo de pensión que tiene un carácter extraordinario. Esta consiste en un complemento a la pensión básica por un valor que se calcula con base en el ahorro que cada persona y su cónyuge hayan realizado a lo largo de su vida laboral. Es de carácter contributivo.

b) Chile: a partir del 2008, con la Ley 20.255, Chile empezó a encaminarse gradualmente hacia un sistema de pilares. Como parte del pilar solidario (pilar cero), diseñó una pensión que se otorga a aquellas personas que no hayan obtenido ningún tipo de pensión, tengan más de 65 años y se encuentren entre el $60 \%$ de la población con menores ingresos. Actualmente se entrega un monto equivalente a USD I37.

Esta pensión se financia con recursos del presupuesto de la nación y, por tanto, es de carácter no contributivo. También tiene un ajuste en función de lo que haya aportado a la seguridad social, siempre 
y cuando no haya logrado una pensión mínima. Se financia con los recursos aportados por los afiliados.

Aunque se piensa desmontarla en el futuro, el sistema aún conserva el esquema de pensión mínima para las personas que, no habiendo podido completar el capital necesario para tener una pensión de salario mínimo, tengan por lo menos un número de semanas cotizadas.

c) Argentina: en este país se cuenta actualmente con un sistema de reparto de carácter contributivo que reemplazó el sistema de pilares que existía desde 1993. Hay una pensión dirigida a las personas mayores de 70 años cuando ni ellas ni sus familias perciben ingresos por pensión o retiro de cualquier régimen. También cubre a las personas con un grado de invalidez superior al $76 \%$. Para las personas mayores de 70 años la pensión es el $70 \%$ de una pensión mínima y para los inválidos del $100 \%$. Este esquema es de carácter no contributivo.

d) Uruguay: el sistema uruguayo cuenta con un pilar solidario. A diferencia de los casos anteriores, es de carácter contributivo, ya que se financia con las cotizaciones de todos los empleados hasta un nivel de salarios (nivel básico).

Es un sistema de reparto en el que se otorga una pensión básica calculada con base en el salario promedio base del afiliado de los últimos ıo años. El sistema de capitalización individual opera para las cotizaciones que se hagan sobre salarios por encima del nivel básico hasta un tope. Está soportado económicamente para el pilar solidario por las cotizaciones del nivel básico y por el aporte de los empleadores de todos los niveles de salario para el complementario.

Existe además una pensión asistencial de aproximadamente USD I5O que cobija a aquellas personas que, llegadas a la edad de los 70 años, no tienen ingresos o, de tenerlos, son inferiores al monto de la pensión.

e) Bolivia: su sistema tiene dos componentes solidarios. El primero se llama Renta Dignidad y se entrega a todas las personas que superan los 60 años, sin requisitos adicionales. Es de carácter no contributivo y consiste en un pago equivalente de USD 36 para aquellas que no tienen pensión del régimen contributivo y de USD 29 para los que sí la tienen.

En lo referente al régimen contributivo, hay una pensión llamada "de solidaridad", que opera en los casos en que llegado a la edad, el capital acumulado no le permite al afiliado obtener una pensión por encima del $60 \%$ del salario promedio de cotización. Este esquema tiene una financiación mixta que incluye aportes y recursos estatales. 
f) Brasil: existen dos esquemas de protección para las personas que no han logrado tener una pensión. El primero es un programa llamado Beneficio de Protección Continua que cubre a aquellas personas que no reciben pensiones y que hacen parte de familias con ingresos inferiores al $25 \%$ del salario mínimo. La prestación es el equivalente a un salario mínimo y lo reciben las personas mayores a 65 años o que están discapacitados y en extrema pobreza. Es de carácter no contributivo

El segundo, llamado Pensión Rural está dirigido a la población rural informal. Es un ingreso que se entrega a los adultos hombres con edades por encima de los 60 años y mujeres mayores a 50 años, por un valor equivalente a un salario mínimo. Es financiado con recursos especiales del presupuesto del Estado.

\section{Definición de LoS tipos de PEnSiones eSEnCiales}

Con base en los casos descritos, se propone, a continuación, una tipificación de los esquemas de pensión esencial existentes, con base en las características principales que comparten.

a) Pensión de sobrevivencia o asistencial: con este término, se denominan aquellas pensiones que otorga el Estado con el cumplimiento de criterios mínimos como: superar una edad determinada, ser parte de un sector vulnerable de la población y no contar con ingresos individuales o familiares o que estos no superen la línea de pobreza o de indigencia.

Estas pensiones corresponden a un porcentaje de un salario mínimo o del salario promedio del país, buscan cubrir a la población mayor de 60, 65 o 70 años, dependiendo del caso, y apuntan a superar la línea de pobreza.

Se trata de pensiones no contributivas que generalmente son financiadas con recursos del Estado. Los sistemas de Dinamarca y Chile incluyen esquemas de estas características.

b) Pensión mínima garantizada: se basa fundamentalmente en la reforma chilena de principios de i980 que creó el régimen de ahorro individual. Es de tipo contributivo y financiado con una parte de los aportes de los afiliados al régimen. Dadas unas condiciones mínimas, pero exigentes de edad y semanas cotizadas, el Estado garantiza a los afiliados el pago de una pensión definida, equivalente en muchos casos a un salario mínimo, así el capital ahorrado individualmente no sea suficiente para obtener esa pensión. 
c) Pensión básica o social: es un tipo de pensión que consiste en que la persona recibe una pensión cuyo monto se define con base en el salario. Esta puede ser complementada por otra pensión que se determina en función del capital que logren ahorrar la persona y su cónyuge, en su vida laboral.

Los sistemas de Dinamarca y Uruguay incluyen pensiones de estas características.

d) Planes voluntarios de ahorro con subsidio del Estado: se trata de un sistema en el que la persona ahorra de acuerdo con su ingreso y logra reunir un capital que luego es complementado con un subsidio del Estado.

Al llegar a una edad determinada, la persona puede recibir un ingreso mensual que se calcula teniendo en cuenta ese ahorro alcanzado y la expectativa de vida. Es un sistema mixto, en el que se combina un esquema contributivo voluntario y un subsidio no contributivo por parte del Estado.

El caso de los beps en Colombia, al cual nos referiremos más adelante, es un ejemplo de este tipo de esquema.

Visto desde el punto de vista de un sistema de pilares, estos tipos de pensión asistencial terminan en últimas siendo parte de un pilar solidario conocido también como pilar cero y en el que existen dos modalidades:

La primera se trata de un ingreso mínimo de subsistencia fondeado con recursos del Estado (que se otorga de forma universal). En estos casos de carácter no contributivo.

La segunda es una pensión igual o básica para todos los afiliados al sistema, financiada con los aportes de dichas personas, calculada como una proporción de sus ingresos. Esta es de carácter contributivo.

El esquema actual chileno es un ejemplo de este tipo de esquema.

\section{El CaSO COLOMbiano: consideraciones Para la IMPLEMENTACIÓN DE UN ESQUEMA DE PENSIÓN ESENCIAL}

\section{Aproximaciones a la pensión eSEnCial en el Sistema ACtual COLOMBiano}

El sistema pensional actual colombiano, establecido mediante la Ley roo de I993 y posteriores reformas, ha incluido algunos de los aspectos ya mencionados en relación con los esquemas de pensión esencial.

En dicha reforma, se estableció en Colombia un esquema de pensión mínima garantizada de un salario mínimo para aquellas personas que pudieran 
cotizar y que cumplieran con un mínimo de exigencias. Para acceder a ella se estableció como requisito contar con un mínimo de semanas de cotización, que varía entre el régimen de prima media y el de capitalización individual. Los recursos para pagar estas pensiones provienen de tres fuentes: (i) cotizaciones de todos los afiliados y sus empleadores, (ii) subsidios dados por el Estado para cubrir el faltante de los afiliados del régimen de prima media y (iii) un porcentaje de las cotizaciones de los afiliados al régimen de ahorro individual que van a un fondo de garantía de pensión mínima. Si bien este esquema es en su mayoría contributivo, también se financia a partir de las reservas del sistema de prima media y, en caso de faltar recursos, del presupuesto de la nación.

También se creó un Fondo de Solidaridad con el fin de implementar el pago de un auxilio al anciano indigente (Programa Colombia Mayor), cuyo monto ha variado en el tiempo y hoy está entre los USD I4 y USD 25 mensuales, dependiendo de los aportes que hagan los municipios (los recursos provienen de aportes adicionales hechos por las personas de más altos ingresos entre el I \% y el $2 \%$ dependiendo del salario y de los recursos de los municipios, en los casos correspondientes), por lo cual puede considerarse como una pensión asistencial que, a diferencia de lo modelos vistos en otros países, es de carácter contributivo, salvo en los casos en que hay una adición del municipio, lo que la haría mixta. Cubre a un grupo de personas limitado y las condiciones para acceder y el número de personas que pueden aplicar a este beneficio, lo define el gobierno.

Más adelante, en 2009, se creó un programa llamado Beps (beneficios económicos periódicos), dirigido a quienes tienen ingresos mensuales inferiores a un salario mínimo. A través de este mecanismo, estas personas tienen la oportunidad de hacer un ahorro que, al llegar a la edad de pensión definida en el RPM, es complementado con un subsidio del Estado por un valor correspondiente al $20 \%$ del monto acumulado y les permite tener un ingreso mensual en función del monto ahorrado. Es de carácter voluntario y contributivo, en la parte ahorrada por la persona. A este sistema pueden acudir aquellas personas afiliadas al R Pм о RAIs que devengan en promedio mensualmente menos de un salario mínimo y que quieran tener un ingreso periódico al llegar a la vejez.

Como se puede ver en los diferentes esquemas mencionados en esta sección, ha habido diferentes iniciativas aisladas para tratar que las personas puedan contar con un ingreso al llegar a la vejez. Sin embargo, consideramos que esto solo se puede lograr si se integran los esquemas contributivos y no contributivos en un solo sistema, que podría ser uno de pilares y dentro de este incluir el concepto de pensión esencial que a continuación desarrollaremos. 


\section{Características básicas de la Pensión esencial}

En un sistema de pilares, siempre se considera indispensable la creación de un pilar básico que tiene como objetivo que todas las personas tengan un ingreso que les permita hacer frente a sus necesidades básicas. Algunos países han optado por garantizar una pensión mínima, con subsidio del Estado, que premie el esfuerzo de las personas que han hecho cotizaciones por un tiempo mínimo determinado, a lo largo de su vida laboral. En otros, se ha separado el sistema contributivo del asistencial. En el primero, la persona tiene derecho a una pensión, siempre y cuando haga un mínimo de cotizaciones. En el segundo, el Estado provee un ingreso de sobrevivencia, con cargo al presupuesto, a aquellas personas que, al alcanzar una edad determinada, no hayan podido crear un patrimonio, una renta o una pensión que les permita cubrir sus necesidades básicas. Tiene por finalidad evitar que esta población quede en estado de indigencia al no poder trabajar.

Si se siguen los lineamientos del punto anterior y ante la necesidad de hacer una reforma pensional, sería necesario determinar qué tipo de esquema funcionaría mejor en Colombia y que haría que la carga fiscal fuera manejable. Para ello, hay aspectos que deben ser evaluados en profundidad. A continuación, se plantean algunas inquietudes o premisas que permitan analizar y concluir cuáles son algunos de los aspectos críticos a tener en cuenta en esta discusión.

\section{Principales características de la Pensión esencial}

La pensión esencial debe ser de carácter universal. Manteniendo la premisa de que una reforma debe como principio básico la protección integral en la vejez es necesario contemplar que, independientemente del monto o las condiciones para llegar a ella (a lo cual nos referimos más adelante), la pensión esencial es un beneficio que debe cubrir a todas las personas que no logren tener una pensión igual o superior a un salario mínimo. Asimismo, debe tener especial énfasis en aquellas que, al llegar a determinada edad, no posean a nivel individual o familiar, un ingreso que supere la línea de pobreza o indigencia. En un documento de Fedesarrollo² que se menciona más adelante y en uno reciente

2 Leonardo Villar, Alejandro Becerra y David Forero. Propuesta y estimación de los costos económicos de extender la cobertura del sistema pensional colombiano, Informe Final presentado a Colpensiones (Contrato n. ${ }^{\circ}$ 85-20r6) (Bogotá: Fedesarrollo, 20I7). https://www.repository.fedesarrollo.org.co/bitstream/handle/I I 445/3447/Repor_Marzo_20I7_Villar_Becerra_y_Forero. pdf? sequence $=3$ \& is Allowed $=y$ 
del Banco Interamericano de Desarrollo3 ${ }^{3}$, se describe la forma en que muchos países de Latinoamérica han implementado esquemas de pensión esencial de acuerdo con sus posibilidades.

En línea con lo anterior, es indispensable que el esfuerzo por crear un esquema de cobertura universal tenga en cuenta las características y necesidades particulares de los trabajadores, especialmente considerando el alto grado de informalidad existente, especialmente en el sector rural y algunas regiones del país.

Adicionalmente, es indispensable definir cuáles serían los requisitos para acceder a esta pensión. En la mayoría de los países se utiliza únicamente un criterio de edad.

Debe incluir un componente de pensión básica y un ajuste complementario. $\mathrm{El}$ primero de estos corresponde a un valor mínimo que cubra las necesidades básicas de una persona y debe lograr que el mayor número de ellas, al llegar a los 65 años, tenga un ingreso que supere la línea de pobreza o de indigencia. El acceso a esta pensión no exigiría requisitos de cotización, sino haber llegado a una edad determinada y no tener ingresos suficientes

Esta pensión debería tener un complemento con un tope equivalente a un porcentaje del salario mínimo que se definiría a partir de las semanas cotizadas al sistema formal o ahorradas de forma voluntaria a través de los BEPS. De esta manera, el acceso a la pensión básica y al ajuste complementario, se entregarían en lugar de la devolución de saldos, en el RAIs, de la indemnización sustitutiva, en el RPM, cuando las personas tengan derecho a ella o del beneficio logrado a través de los Beps. Todo lo anterior conduciría a la integración de estos mecanismos.

En este mismo sentido, Fedesarrollo ${ }^{4}$ plantea que a través de los BEPs se podría estructurar este beneficio, siempre y cuando se amplíe su cobertura a todas las personas que voluntaria u obligatoriamente contribuyan, pero que no puedan cumplir los requisitos para tener una pensión de un salario mínimo.

La financiación de un esquema de estas características debe ser mixta. En muchos casos la decisión sobre si una pensión es contributiva o no está definida por la población objetivo. En este sentido, suele determinarse que la prestación sea contributiva si hace parte de un sistema formal de seguridad social y cubre a las personas que, habiendo cumplido con los requisitos de edad y semanas

3 Álvaro Altamirano Montoya, Solange Berstein, Mariano Bosch, Manuel García Huitrón y María Laura Oliveri, Presente y futuro de las pensiones en América Latina y el Caribe, Monografía 649 (Banco Interamericano de Desarrollo, 20I8). https://publications.iadb.org/publications/spanish/ document/Monografia_Presente_y_Futuro_de_las_Pensiones_en_ALc.pdf

4 Villar, Becerra y Forero, Propuesta y estimación de costos económicos, 2017. 
cotizadas, no tienen el capital necesario para tener una pensión equivalente a un salario mínimo. En el caso opuesto, en los sistemas de reparto o de pilares, la pensión básica que se otorga está dirigida a cubrir un nivel de subsistencia y se dirige a las personas más necesitadas o a toda la población.

Bajo el esquema propuesto, que incluye un ingreso básico de subsistencia para todos, sin requisitos de cotización, y un ingreso complementario, graduado por el nivel de cotizaciones o ahorro, la financiación del esquema sería mixta (no contributiva para la pensión básica y contributiva para el ajuste complementario). En este sentido, los fondos provendrían de recursos del Estado, con ahorros o reservas provenientes de las cotizaciones y utilizando las fuentes actuales provenientes del Fondo de Solidaridad, Fondo de Garantía de Pensión Mínima, Aportes de los Pensionados, así como los subsidios que hoy se les dan a las pensiones de mayores ingresos. En caso de establecerse un impuesto a las pensiones, estos recursos deberían dedicarse a ser parte de esta financiación.

Un ejemplo de esto es el caso de Chile en el que el total de la pensión, llamada social, es la suma de una pensión básica universal (рвs) y un factor adicional (APS). Este último es un factor que hace que la pensión total se incremente a medida que hay un mayor esfuerzo por cotizar. Habría que crear una tabla de factores para determinar la parte complementaria de pensión en función del tiempo de aporte y la relación con el гвс.

\section{Cambios en los esquemas eXISTENTES}

En el caso de una reforma y especialmente del sistema de pensiones, su aprobación en el Congreso sería más viable si no se cambian las partes esenciales del sistema que está vigente. Sería deseable mantener el esquema de PMG (Pensión Mínima Garantizada), Рсм (Programa Colombia Mayor), вEPs (Beneficio Económico Periódico) y pensión familiar.

Sin embargo, estos mecanismos podrían requerir algunas modificaciones si se quisiera avanzar hacia la implementación de una pensión esencial con las características descritas. A continuación, se presentan algunas consideraciones al respecto:

\section{PMG (Pensión Mínima Garantizada)}

La pensión mínima garantizada es un esquema que tiene su fundamento en un sistema contributivo. Solo cobija a aquellas personas que, a pesar de cumplir unos parámetros establecidos, no pudieron reunir el capital necesario para tener una pensión equivalente a un salario mínimo, por lo cual el Estado o un fondo especial cubren ese faltante. No es de carácter universal y, por tanto, 
no se puede considerar como parte de un sistema integral de protección a la vejez, así lo haga para una parte de la población.

La informalidad laboral en Colombia y la dificultad para mantener un ingreso estable hacen que la densidad de la cotización sea baja e impiden que un número importante de personas pueda acceder a este beneficio.

Las personas que no pueden cumplir con los requisitos tienen la posibilidad de pedir la indemnización sustitutiva en el R Pм o la devolución de saldos en el rais. Aunque puede ser un valor importante, tiene un riesgo grande de ser utilizado para otros fines y de que no ayude a tener un ingreso estable para la vejez.

No se estima que este mecanismo haya tenido efecto significativo para lograr que haya personas mayores de 60 años con una pensión. Aún así, es importante para aquellos que tienen un empleo formal y que hacen parte del grupo que puede cumplir con los requisitos.

Al dar una tasa de remplazo cercana al Ioo \% tiene un nivel alto de subsidio por parte del Estado en el caso del RPM y de los cotizantes en el caso del RaIs. Esto haría que en caso de lograrse una mayor formalidad en el empleo, el valor total de los subsidios se incrementaría de forma importante.

Al reflexionar sobre estas consideraciones y manteniendo la premisa de que es deseable establecer un sistema que proteja integralmente a las personas en su vejez, se hace necesario que el sistema que opere deba ser de carácter universal y que cubra a las personas que hayan podido contribuir en forma continua, en forma parcial o no lo hayan podido hacer. Una pensión esencial debería remplazar una pensión mínima, pero teniendo en cuenta los siguientes aspectos:

I. Debe haber una diferencia entre la pensión que obtenga la persona que nunca cotizó y la que sí lo hizo. Debe haber un factor de gradualidad que permita que quien cotice unos parámetros mínimos tenga una pensión igual o similar a un porcentaje del salario mínimo o del salario promedio del país. Este esfuerzo de cotizar debe ser premiado, así sea de forma obligatoria o voluntaria.

2. Debe haber diferenciación entre la pensión en el sector rural y el urbano, dependiendo de los niveles de subsistencia básica de cada contexto.

Las tendencias de comportamiento en el futuro, en la formalidad del empleo, el crecimiento de la población mayor de 65 años, la natalidad y otros cambios demográficos y factores, deben ser la base para determinar el nivel y la gradualidad de la pensión básica. 


\section{Programa Colombia Mayor (PCM)}

A diferencia de la PMG, al Plan de Adulto Mayor se le podría dar un mayor alcance, buscando que el monto entregado fuera igual o superior al ingreso de pobreza o de indigencia.

Tener un nivel básico para toda la población convertiría el Pсм en un sistema de carácter universal. De acuerdo con esto, el reto más grande será lograr que todos los que tienen ingresos de cualquier tipo coticen de manera obligatoria o voluntaria. Será necesario estimular la cotización, controlando también de manera eficaz la evasión. Corresponderá analizarse si debería haber diferencia entre el sector rural y urbano.

La conversión a una pensión básica solo sería posible si se elimina la restricción constitucional que existe de que ninguna pensión en Colombia puede ser inferior a un salario mínimo, salvo que se acogiera lo planteado en su documento por Fedesarrollo de hacerlo usando el esquema de los Beps.

\section{$B E P S$}

Los cambios en la estructura de los Beps no deberían ser importantes, si se les considera como un elemento que incrementa la pensión básica. Sería necesario determinar la forma en que se mantendría el subsidio del Estado. Este podrá definirse a través de la gradualidad del ajuste complementario de la pensión básica o como un porcentaje de la cotización hecha por el afiliado al sistema, dado que a diferencia del esquema contributivo, no tiene el soporte de la proporción que aporta el empleador, según cálculos realizados por Alejandro Nieto de la Universidad Externado de Colombia, una persona cotizando I300 semanas y con una tasa real del $4 \%$, acumularía un capital de cerca de \$ro9 millones, mientras que en el mismo periodo una persona en BEPs y con una tasa real del $3 \%$, tan solo acumularía \$2 8 millones $^{5}$.

\section{Pensión familiar}

El fundamento de la creación de la pensión familiar en Colombia es la oportunidad que se les debe dar a las parejas de tener una pensión conjunta, cuando individualmente no reúnan los requisitos para obtenerla. Para mantenerla, sería pertinente tener presentes las siguientes consideraciones: 
Según las normas actuales, en el RaIs acceder a esta pensión está condicionado que la pareja logre un capital conjunto que le permita tener una pensión igual o superior al salario mínimo o que cumplan con los requisitos para tener una pensión de salario mínimo. El subsidio, claramente estará en la diferencia entre el capital ahorrado y el necesario para financiar una pensión de estas características.

En el régimen de prima media, esta opción se encuentra limitada a aquellas personas que hacen parte del Sisbén i y 2. Debería ampliarse para cualquier pareja que haga parte del régimen y así poder ser equiparado con el RAIs.

Aquellas personas que se acojan a este tipo de pensión no deberían tener acceso a la pensión básica aunque mantendrían la posibilidad de optar por ella de manera individual, en cuyo caso, el esfuerzo fiscal sería mayor.

\section{a. Viabilidad del establecimiento de una pensión básica}

Un tema importante por resolver es si los recursos existentes son suficientes o puede implicar un mayor esfuerzo fiscal. En el estudio Propuesta y estimación de costos de extender la cobertura del sistema pensional colombiano ${ }^{6}$, Fedesarrollo plantea cinco alternativas de la cobertura y monto de la pensión básica que van desde mantener la cobertura y el monto actual del "Programa Colombia Mayor" hasta una cobertura generosa de toda la población mayor, donde se pagaría una pensión equivalente al ingreso del nivel de pobreza (ver cuadro 2).

\section{Cuadro 2.}

\section{EsCENARIOS DE SIMULACIÓN}

\begin{tabular}{lll}
\hline & \multicolumn{1}{c}{ Tipo de cobertura } & \multicolumn{1}{c}{ Monto del Subsidio } \\
\hline o. Base - Status Quo & Actual - Colombia mayor & Actual -Colombia mayor \\
\hline I. Atenuación de pobreza & Debajo de línea de pobreza & Línea de indigencia \\
\hline $\begin{array}{l}\text { 2. Complemento pilar contribu- } \\
\text { tivo - Asistencial }\end{array}$ & $\begin{array}{l}\text { Población sin pensión } \\
\text { contributiva }\end{array}$ & Línea de indigencia \\
\hline $\begin{array}{l}\text { 3. Complemento Pilar contribu- } \\
\text { tivo - Generoso }\end{array}$ & $\begin{array}{l}\text { Población sin pensión } \\
\text { contributiva }\end{array}$ & Línea de pobreza \\
\hline $\begin{array}{l}\text { 4. Cubrimiento total - Asisten- } \\
\text { cial }\end{array}$ & Total población mayor & Línea de indigencia \\
\hline 5. Cubrimiento Total-General & Total población mayor & Línea de pobreza \\
\hline
\end{tabular}

Fuente: Fedesarrollo, 2017, p. 99.

6 Villar, Becerra y Forero, Propuesta y estimación de costos económicos, 2017. 
En términos del PIB, en los análisis hechos por Fedesarrollo (ver cuadro 3), la primera opción que es la menos onerosa, costó o,I3 del piв en el 2016 y costaría $0,38 \%$ del PIB en el 2050 , y en la más generosa hubiera representado de tenerse el I,57 \% del pib en el 20r6 y representaría el 2,95\% en el 2050. Sin embargo, habría alternativas que, siendo menos generosas, estarían en niveles promedio de los países de Latinoamérica. En los lugares donde se han creado, este tipo de pensiones representan entre el o,4 \% y el г \% del piв.

\section{GráfICO 2.}

\section{Costo total del pilar no contributivo ante} ESCENARIOS DE MONTO Y COBERTURA

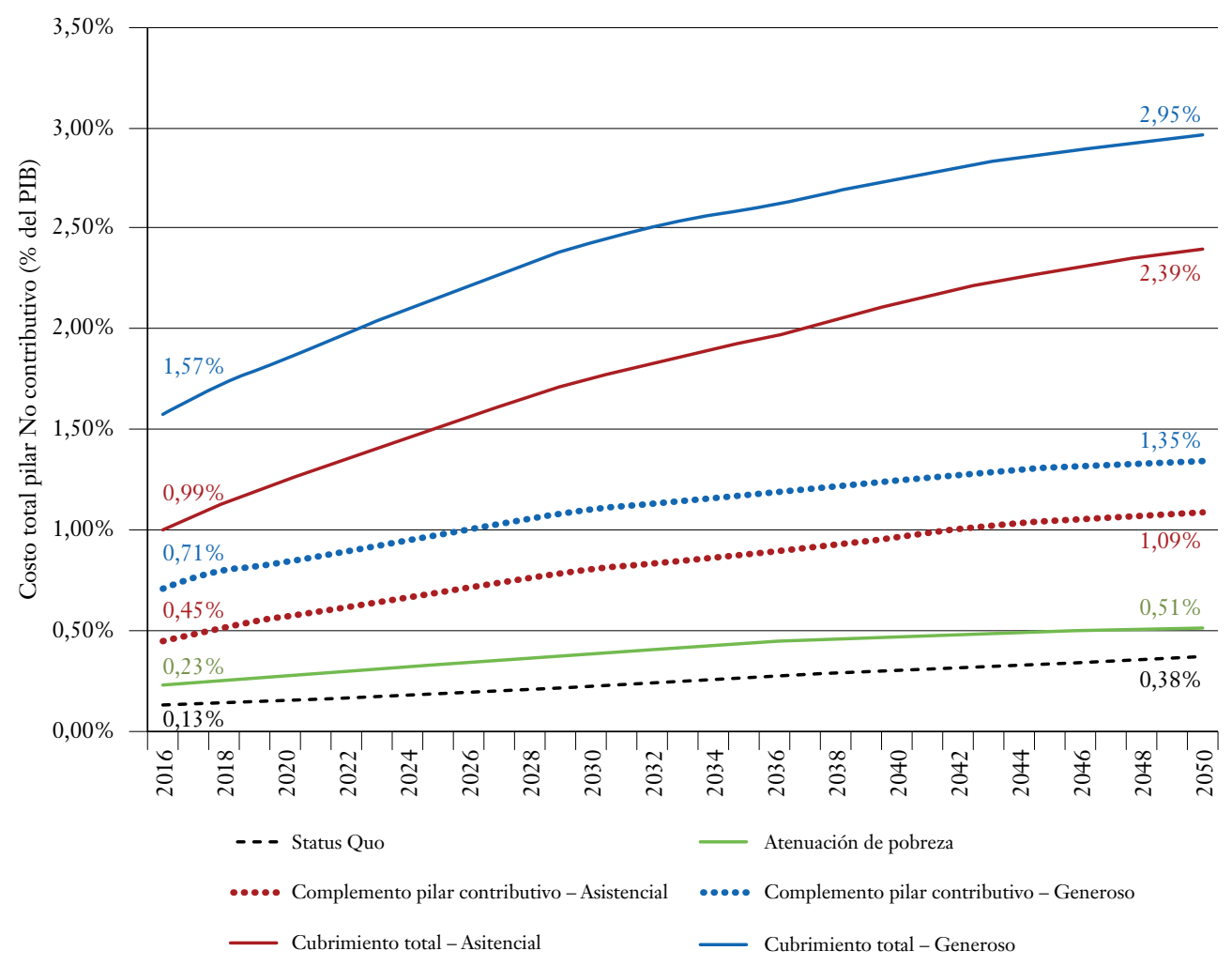

Fuente: Fedesarrollo, 2017, p. Iоo.

Los países que han implementado pensiones esenciales han mostrado resultados positivos en la reducción de la pobreza dentro de la población mayor de 65 años. Como se ha mencionado, Colombia presenta uno de los escenarios más críticos en relación con esta problemática, comparado con el resto de América Latina. Por esto, se esperaría que un esquema de estas características tuviera 
resultados importantes en disminución de pobreza y un efecto significativo en el aumento del consumo de los hogares.

Este esfuerzo debe estar acompañado de un aumento considerable en la formalidad laboral, control de la evasión y en acciones decididas para que en el futuro cada vez sea menor el número de personas que llegan a los 65 años sin recursos para que puedan lograr su sustento.

De igual forma, se requiere un cambio en la distribución del gasto de pensiones que se hace en Colombia contra el presupuesto nacional. Modificar los regímenes especiales que aún subsisten sería importante en el fondeo de las pensiones esenciales

Los aspectos mencionados anteriormente son de particular relevancia si se tiene en cuenta que las tendencias demográficas mundiales indican que en las próximas décadas habrá un crecimiento significativo de la población mayor a 65 años. En este escenario, el esfuerzo fiscal necesario para implementar estos sistemas podría ser mayor a lo proyectado y sería indispensable implementar medidas que incentiven la cotización para lograr que el índice de adultos mayores mantenga o reduzca su participación frente a la población que cotiza.

\section{b. Integración de los recursos de los municipios}

Se tiene que buscar la forma en que los recursos que hoy aportan los municipios para dar un mayor ingreso en el Programa Colombia mayor puedan ser integrados al esquema integral, manteniendo el beneficio para las personas que viven en esos municipios.

\section{c. Cambios necesarios en la administración y supervisión del sistema y de los agentes}

La posibilidad de desarrollar un nuevo pilar que hoy no existe o existe parcialmente implica cambios en el esquema de administración. Es crítico que el diseño del sistema que empezaría a regir después de realizada la reforma esté muy bien estructurado. En relación con esto se plantean las siguientes consideraciones:

- Es necesario lograr la integración de la administración del sistema. Para el esquema que acá se propone la estructura sombrilla debería ser un sistema de pilares donde la pensión esencial sea parte del pilar o o I de carácter universal. Como ya se ha mencionado, tendría un componente universal y no contributivo (pensión básica) y uno complementario contributivo. 
- Podría haber competencia de los administradores para el componente complementario de la pensión esencial o los pilares 2 y 3 (actuales). El componente de pensión básica, en su parte asistencial, debería poder estar a cargo de las administradoras de los demás pilares y de una entidad especializada del gobierno.

- Sería necesario considerar cambios en los procesos de administración frente a los nuevos alcances del sistema. Esto incluiría: la forma en que será cubierta la población rural, incluyendo afiliados y empleadores, el manejo de los procesos de afiliación y recaudo, educación, atención al usuario y pago de la pensión, entre otros.

- Los beps podrían hacer parte del factor complementario de la pensión básica y podrían ser manejados por cualquier administradora pública o privada.

- Se requeriría el desarrollo de una supervisión más especializada en el sector.

- Podría mantenerse el subsidio de la cotización para invalidez y sobrevivencia en el caso de los BEPS.

\section{d. Es necesario desarrollar la educación a los afiliados sobre el sistema}

En países como Chile, las reformas y ajustes en ellas han incluido la destinación de recursos y esfuerzos dirigidos a que el mayor número de personas tengan un conocimiento adecuado sobre el sistema de pensiones. El Estado colombiano no ha implementado acciones importantes en este sentido. Una reforma pensional sería un buen escenario para trabajar en ello, buscando que la población conozca, al menos, los siguientes aspectos:

- Diferentes pensiones a las que se pueden acceder y cómo obtenerlas.

- Esquemas de contribución y de ahorro.

- El efecto que las tasas reales pueden llegar a tener en el logro de una pensión y la potencial tasa de reemplazo.

- La importancia de cotizar y de hacerlo lo más regularmente posible, así sea de manera voluntaria.

- En caso de establecerse una pensión esencial, información sobre los derechos que se tienen, cómo acceder a ellos, los efectos positivos de hacer cotizaciones, entre otros.

- Derechos a pensión de invalidez o sobrevivencia y cómo acceder en caso de siniestro.

- Relación entre el ahorro y la pensión esencial y entre esta y los sistemas contributivos. 
- Qué hacer si no se pueden cumplir los parámetros para obtener una pensión mínima y opciones para convertir la devolución de saldo o indemnización sustitutiva en pensión o BEPs.

\section{e. Otras consideraciones a tener en cuenta}

Hay una serie de consideraciones que se debería estudiar a mayor profundidad, en caso de hacer una reforma. Se presentan aquí únicamente de manera enunciativa:

- Crear una pensión esencial de carácter universal conlleva la necesidad de determinar la forma en que se cubriría y por parte de quien el aporte a la salud de aquellas personas que no hagan parte del Sisbén.

- Incentivar a las personas para que coticen o hagan un ahorro que les permita tener un ingreso cuando no puedan trabajar y se reduzca la carga al Estado. Esto especialmente para aquellas independientes que teniendo ingresos suficientes no cotizan y sí se beneficiarían en el caso de crearse una pensión esencial.

- Fomentar la cotización voluntaria asumiendo por parte del Estado, parcial o totalmente las cotizaciones para el seguro de invalidez y sobrevivencia.

- Incentivar el trabajo social con las poblaciones vulnerables, incluido el sector rural, en todos los aspectos que permitan mejorar el acceso y la utilización de los esquemas de seguridad social y el conocimiento de los derechos y las obligaciones.

- El Estado debe considerar cómo se puede mantener el tejido social de las familias y la solidaridad en ellas sobre todo para la población que llega a la vejez y definir cuál es el rol para seguir en el futuro, directamente o indirectamente. Además debería evaluarse, dada su cobertura y objeto social, el papel que las cajas de compensación pueden llegar a desempeñar, en aquellos aspectos que tienen que ver con la calidad de vida del adulto mayor, tal como algunas de ellas lo han venido haciendo.

- Definir cómo enfrentar los retos en la protección integral y en el papel que desempeña el Estado frente a cambios demográficos relacionados con el incremento más que proporcional de la población mayor, el bajo índice de natalidad, la extralongevidad y todos los aspectos relacionados con la perdida paulatina del bono demográfico. 


\section{CONCLUSIONES}

Aunque en el documento se analizan varios aspectos y se tienen en cuenta diferentes alternativas, esta sección de conclusiones se limitará a resumir los aspectos principales que consideramos deben tenerse en cuenta para próximas discusiones en torno a una posible reforma pensional en Colombia.

a) La reforma que se vaya a estudiar debe tener un énfasis en la integralidad de esta en función de la protección a las personas cuando llegan a la vejez.

b) La estructura del sistema debe ser de pilares donde claramente se desarrolle un pilar o o I que incluya una pensión básica de subsistencia (que cubra nivel de indigencia o pobreza) y un ajuste complementario que este determinado con base en el nivel de cotizaciones (contributivo) que pueda hacer la persona de forma obligatoria, con apoyo de su empleador, o de forma voluntaria (BEPs o fondos voluntarios).

c) El desarrollo de cualquier esquema debe incluir consideraciones para la cobertura de la población rural.

d) Diferentes países han desarrollado una pensión esencial de carácter generalmente asistencial (no contributivo) aunque hay algunos de ellos como Chile y Dinamarca que lo han hecho de forma mixta con un componente asistencial (no contributivo) y un ajuste complementario (contributivo). En términos promedio los costos de estos programas están cercanos al i \% del piB, aunque en algunos países como Brasil están más por el orden del $7 \%$ del рів.

e) Las características de una pensión esencial deberían ser:

- De carácter universal y que cubra a toda la población, incluida la rural.

- Tener un componente básico que cubra el nivel de indigencia o de pobreza y uno complementario en función de las cotizaciones hechas. El monto total debería ser una proporción del salario mínimo o del salario promedio de la población.

- Financiación con recursos del Estado, cambio de destinación de fondos como el FGPm y Fondo de Solidaridad, aportes actuales de los pensionados, disminución de los subsidios a los afiliados de mayores ingresos e impuestos a las pensiones, si se crearan.

- Tratar de mantener los esquemas de PMg, Beps y pensión familiar con una serie de cambios que permitan la integralidad del sistema y su cobertura. 
f) Hay que hacer cambios en la estructura de administración, dado que

- Es un desarrollo de un esquema de pilares que da un mayor alcance e integralidad a su administración.

- Se requiere la integración de todos los pilares en un solo sistema.

- Es un esquema de competencia entre administradores y no de sistemas.

g) Hay necesidad de hacer un mayor desarrollo en la capacitación de los afiliados sobre el sistema que les permita tener un conocimiento de sus derechos y obligaciones. Esta capacitación debe incluir necesariamente al sector rural.

h) Hay que tener en cuenta unas consideraciones adicionales, tales como:

- Cómo sería la cobertura en salud para los que accedan a la pensión esencial, sea básica o complementaria.

- Cómo se debería incentivar la cotización obligatoria y voluntaria y controlar la evasión.

- Como mantener y promover el tejido social de los ancianos.

- Cómo hacer frente a los cambios demográficos.

- Como crear mecanismos que permitan mejorar la calidad de vida de las personas que llegan a determinada edad.

\section{Bibliografía}

Altamirano Montoya, Álvaro, Solange Berstein, Mariano Bosch, Manuel García Huitrón y María Laura Oliveri. Presente y futuro de las pensiones en América Latina y el Caribe, Monografía 649. Banco Interamericano de Desarrollo, 2018. https://publications.iadb. org/publications/spanish/document/Monografia_Presente_y_Futuro_de_las_Pensiones_en_ALC.pdf

Fedesarrollo y Fundación Saldarriaga Concha. Misión Colombia Envejece: cifras, retos y recomendaciones. Bogotá: Fundación Saldarriaga Concha, 2015. https://www.fedesarrollo.org. co/sites/default/files/envejece.pdf

Nieto, Alejandro. "Colombia: un solo país, múltiples realidades. Una mirada regional del empleo en Colombia, en el marco de una reforma de protección a la vejez". Páginas de Seguridad Social 2, n. ${ }^{\circ} 3$ (2018). https://doi.org/IO.18601/25390406.n4.02

Villar, Leonardo, Alejandro Becerra y David Forero. Propuesta y estimación de los costos económicos de extender la cobertura del sistema pensional colombiano. Informe Final presentado a Colpensiones (Contrato No o85 - 2016). Bogotá: Fedesarrollo, 20I7. https://www. repository.fedesarrollo.org.co/bitstream/handle/I I 445/3447/Repor_Marzo_2OI7_Villar_Becerra_y_Forero.pdf? sequence $=3$ \& isAllowed $=\mathrm{y}$ 